Proc. Vth International Workshop, Advances in Signal Processing for Non Destructive Evaluation of Materials Québec City (Canada),2-4 Aug. 2005. ( X. Maldague ed., É. du CAO (2006), ISBN 2-9809199-0-X

\title{
Reliability Evaluation of NDT Techniques for Cu-Welds for Risk Assessment of Nuclear Waste Encapsulation
}

\author{
Christina Müller ${ }^{(1)}$, Håkan Rydén ${ }^{(2)}$, Mstislav Elagin ${ }^{(1)}$, Carsten Bellon $^{(1)}$, Uwe Ewert $^{(1)}$, Ulf \\ Ronneteg $^{(2)}$, Martina Scharmach ${ }^{(1)}$, Bernhard Redmer ${ }^{(1)}$ \\ ${ }^{(1)}$ BAM - Federal Institute for Materials Research and Testing, Unter den Eichen 87; 12205 \\ Berlin; Germany \\ ${ }^{(2)}$ SKB - Svensk Kärnbränslehantering AB; Oskarshamn; Sweden
}

\section{Keywords}

Deposite, Radioactive Waste, Copper Weldings, Risk Assessment, POD Probability of Detection

friction stir welding and electron beam welding. The quality of the welding process and the reliability of the NDT system

\begin{abstract}
In order to handle the long living radioactive waste (spent nuclear fuel) SKB is planning to build a deep repository that requires no monitoring by future generations. The spent nuclear fuel will be encapsulated in copper canisters consisting of a graphite cast iron insert shielded by an outer 30-50 mm thick copper cylinder for corrosion protection. The most critical part of the encapsulation process is the sealing of the canister, which is done by welding the copper lid to the cylindrical part of the copper shell using - radiographic and ultrasonic testing - must be satisfactorily determined and combined to derive assumptions regarding the frequency of undetected welding defects for the ensemble of canisters as input for the risk assessment. This is done using the POD method according to the "Reliability Handbook MIL 1823" and its generalization to more complex defect situations in welds.
\end{abstract}




\section{The Swedish Deposit Project}

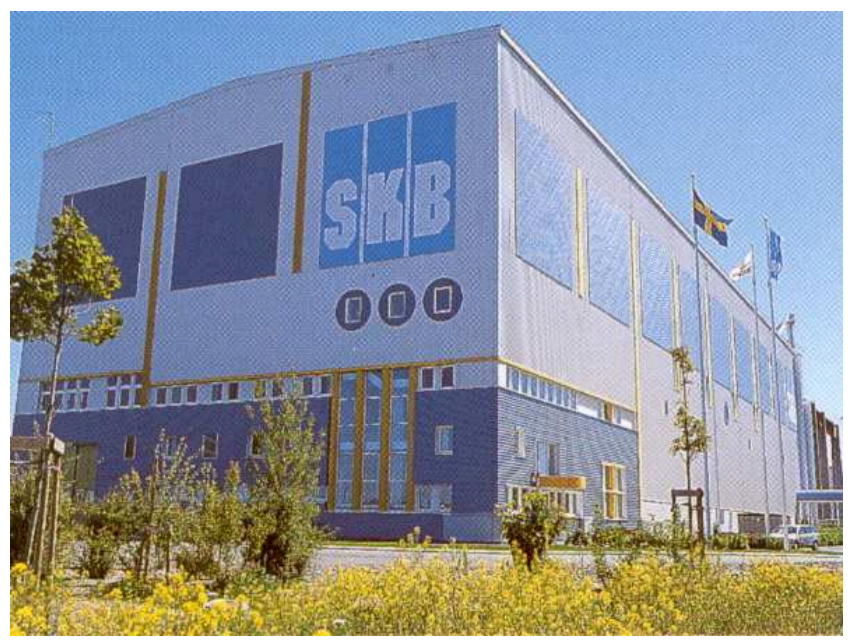

Fig. 1: SKB Canister Laboratory in Oskarshamn

Svensk Kärnbränslehantering AB (SKB, Swedish Nuclear Fuel and Waste Management Co) is responsible for the final disposal of spent nuclear fuel in Sweden.

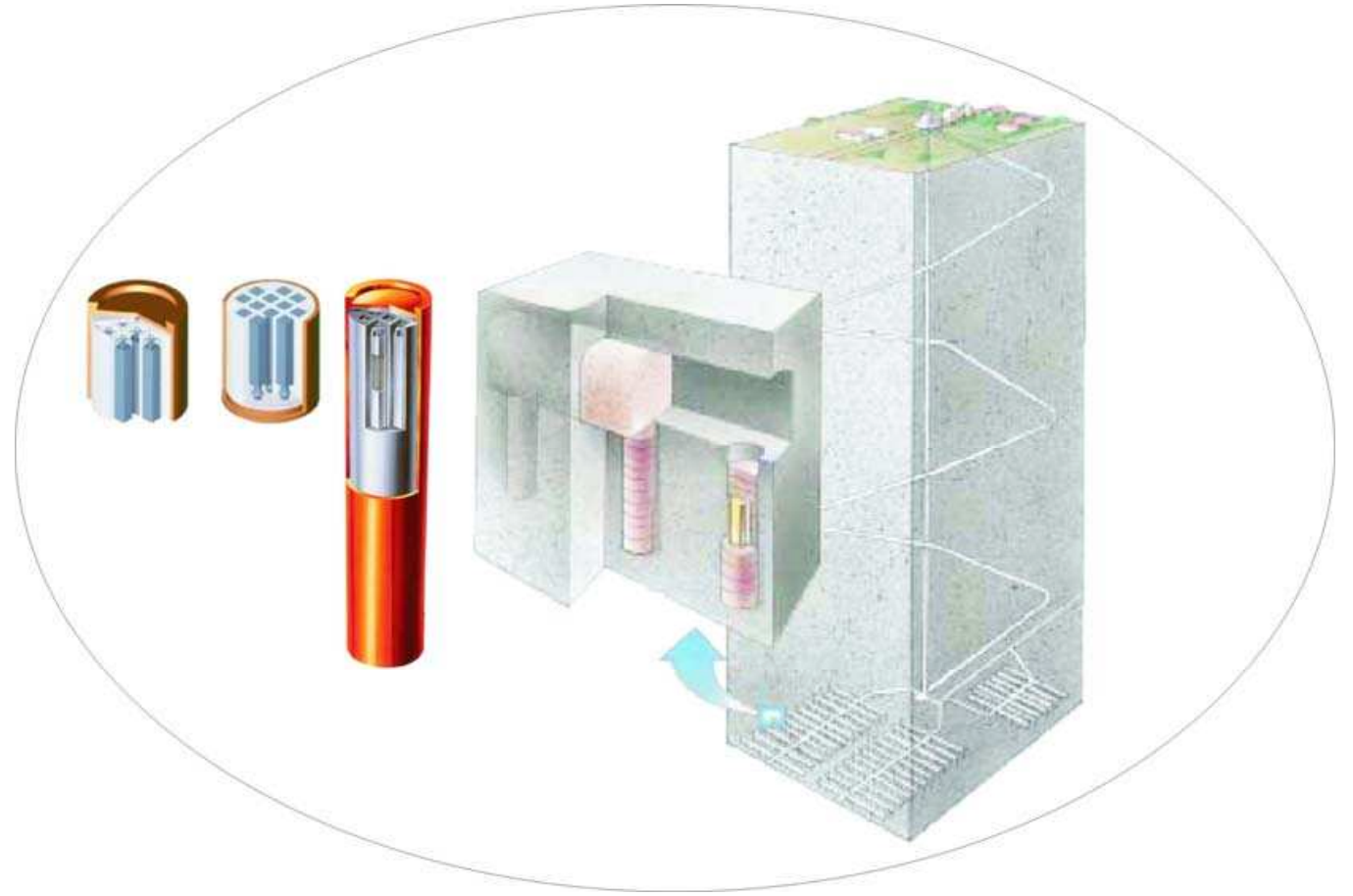

Fig. 2: Construction Plan of Copper Canisters and the Bedrock Deposite 
In order to handle the long living radioactive waste (spent nuclear fuel) SKB is planning to build a deep repository that requires no monitoring by future generations. The spent nuclear fuel will be encapsulated in copper canisters consisting of a graphite cast iron insert shielded by an outer 30-50 mm thick copper cylinder for corrosion protection. The most critical part of the encapsulation process is the sealing of the canister, which is done by welding the copper lid to the cylindrical part of the copper shell. The canisters will then be deposited in the bedrock, embedded in bentonite clay, at a depth of 500 meters.
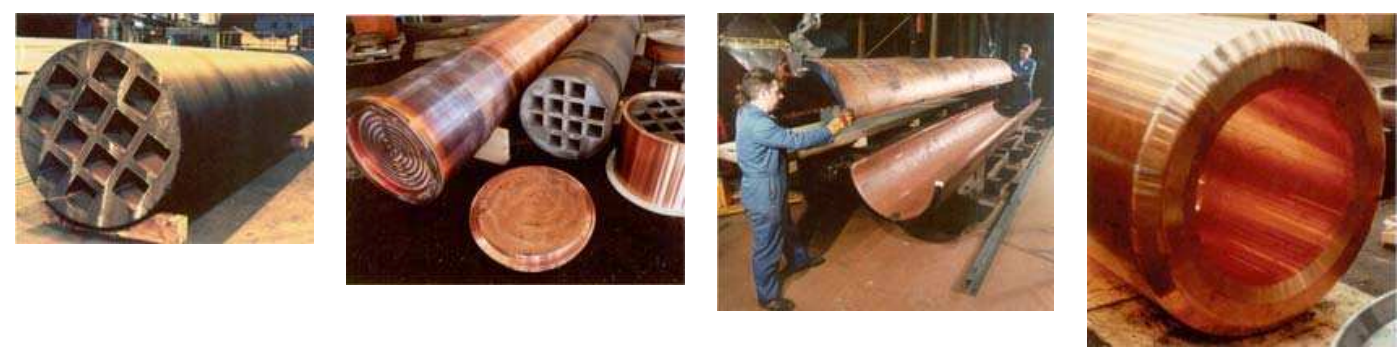

Fig. 3: Construction of the copper canister

The safety and reliability of the whole systems i.e. the well sealed deposit of the radioactive material under all possible influencing scenarios will be guaranteed by modern means of risk management.

\section{Weldings and risk assessment}

The welding techniques studied are electron beam welding (EBW) and friction stir welding (FSW). Both techniques are developed in parallel at the SKB Canister Laboratory (see Fig. 1) in Oskarshamn (Sweden) [1]. According to the different metallurgical welding processes they reveal a quite different variety of defects to be detected by NDT techniques and different material micro-structure. 


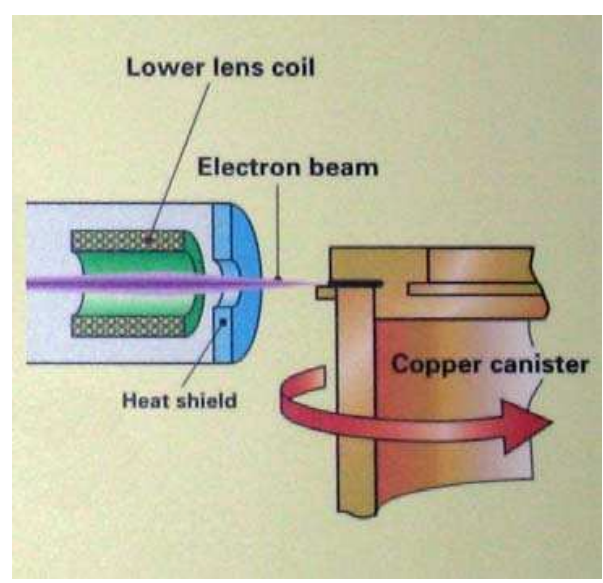

a: Principle of the electron beam welding

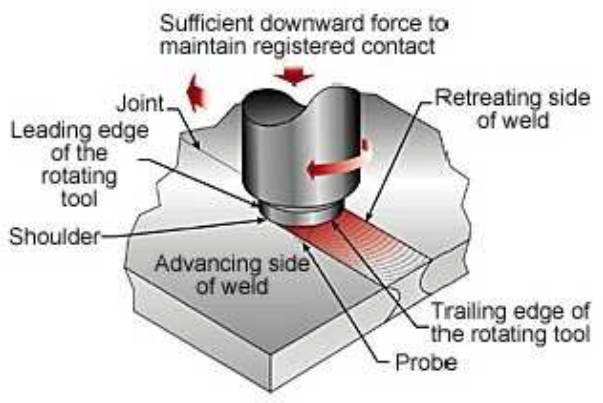

b: Principle of the friction stir welding

Fig. 4: Principle of the electron beam welding and friction stir welding

A subpart of the final risk assessment of the deep repository construction is to determine the risk of premature canister leak caused by defects in the sealing weld. The defects occurring during the production welding process create a diminishing of the wall thickness. The possible additional reduction of the wall thickness by ground water corrosion makes a minimum rest wall thickness of $15 \mathrm{~mm}$ copper necessary. A consequence for the applied NDT methods is to detect all critical defects which would reduce the wall thickness to an amount near or below the $15 \mathrm{~mm}$ with a validated high reliability.

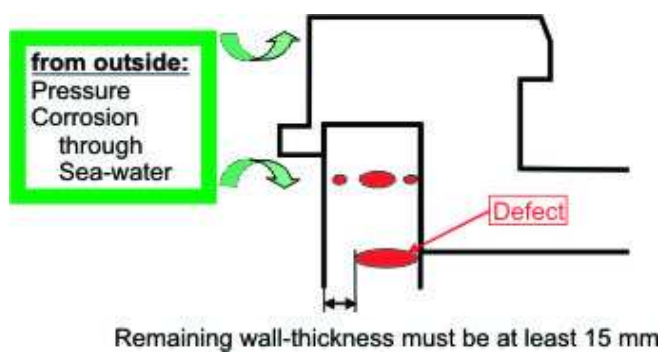

Fig. 5: The weld with possible defect configurations

The NDT techniques applied and adapted by SKB are high energy (9 MeV) X-ray-technique and mechanized ultrasonic phased array technique $(2 \ldots .5 \mathrm{MHz})$ according to material structure [1]. 


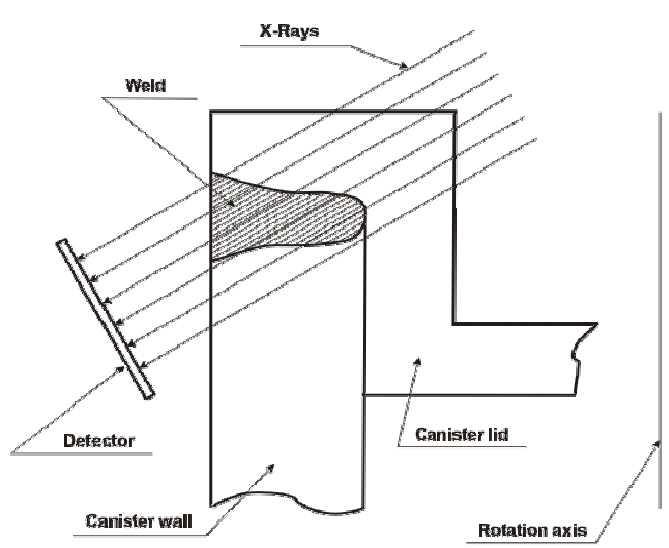

a: Radiographic testing

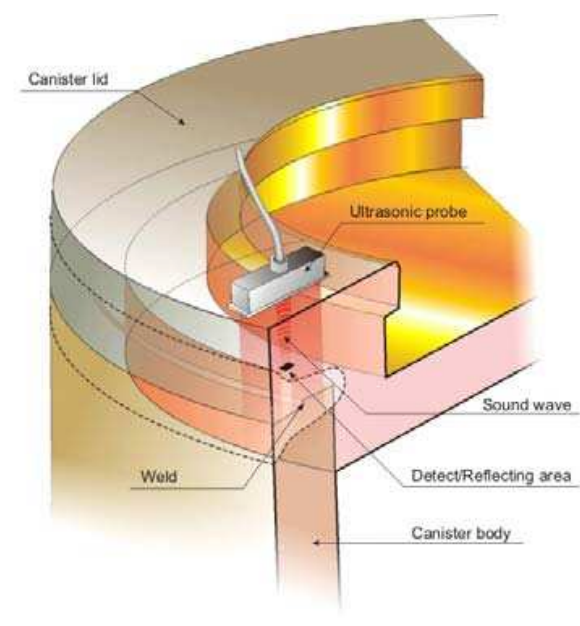

b: Mechanized ultrasonic testing with phased array technique

Fig. 6: Non-destructive testing methods used in the project.

The BAM is providing its expertise in optimizing non-destructive testing and in the corresponding reliability assessment to the project. The applied non-destructive testing methods are in a first step checked and optimized concerning the physical parameters and set up according to the latest European and American standards. For the detailed optimization of the radiographic technique the BAM "Xray" simulation tool [2] is applied to various parameter options thus saving lots of expensive experiments.

\section{Reliability assessment using POD}

Steered by the plan of experiments for the welding procedure optimization and verification, the POD (Probability of Detection) for the defects is determined using a systematic statistical methodology. The POD method, where the detection probability is determined as a function of defect size, was originally developed for the US military aerospace sector [3] for 1-dimensional signals. For the more complex 3-dimensional defect situation in the canister welds and 2-dimensional data fields the method needs to be developed further. From the POD-curve and its lower confidence bound the defect size is derived that will be detected with sufficient reliability and compared to the demand for integrity. This procedure includes series of experiments with the SKB X-ray and ultrasonic methods foreseen for the production. 
Proc. Vth International Workshop, Advances in Signal Processing for Non Destructive Evaluation of Materials Québec City (Canada),2-4 Aug. 2005. @ X. Maldague ed., É. du CAO (2006), ISBN 2-9809199-0-X

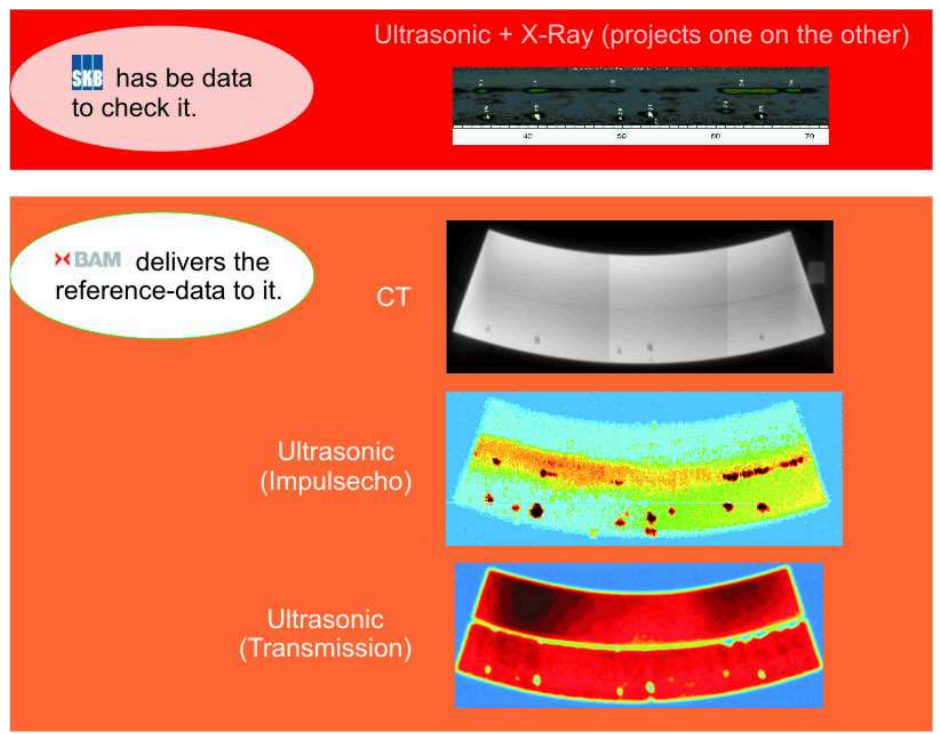

Fig. 7: Example: container L025, section 32-72

These results have to be compared to true defect configurations in the welds. To determine these "true defect configurations" the welds have to be tested destructively or tested with a more comprehensive non-destructive reference method. To save the parts and the effort for the destruction, the BAM selected a high energy computed tomography (HECT, or CT) method as reference completed by focussed ultrasonic transmission measurement.

The basic principle of the signal response analysis or " $a$ versus $a$ " evaluation is shown in

Fig. 8.

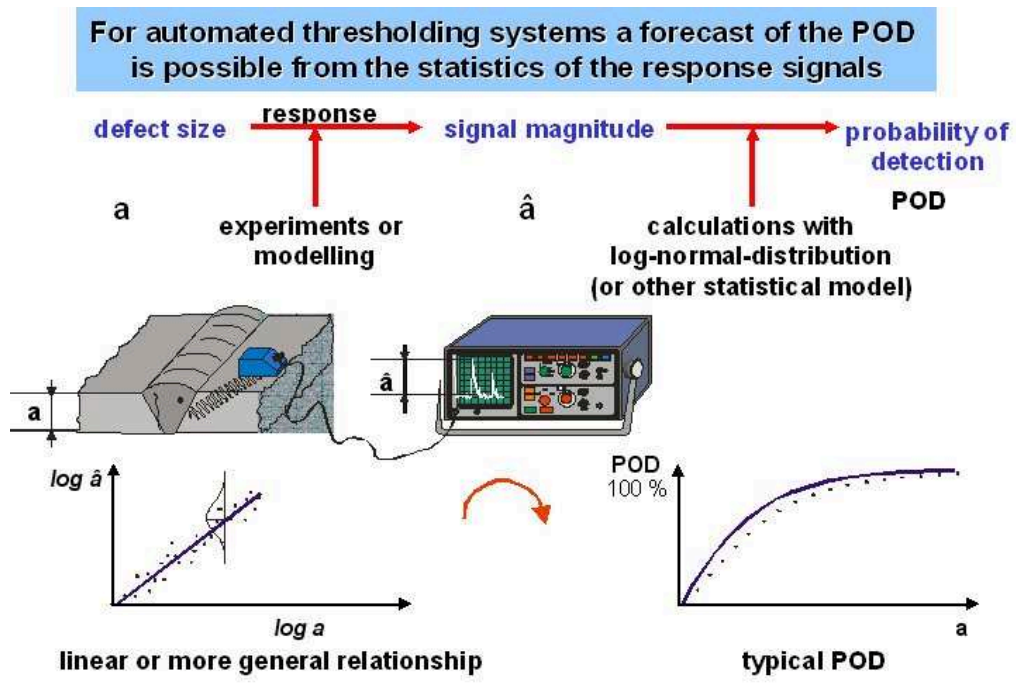

Fig. 8: Quantify the risk: „â versus a“ 


\section{A defect of size $a$ (crack depth in}

Fig. 8) is causing a signal of height $\hat{a}$. The statistical distribution of the signals in dependence of the defect size yields a certain POD curve which is described in more exact terms in the following section.

\section{1 $\hat{a}$ vs. $a$ analysis}

\section{General description [4]}

Consider a quantitative NDT system. As a result of the investigation of a discontinuity having size $a$, it generates a signal $\hat{a}$. If the signal exceeds a certain decision threshold $\hat{a}_{\text {dec }}$ the system registers a flaw detection. As the NDT system is influenced by uncontrolled factors, discontinuities of the same size, can cause signals of different strength. For this reason the strength of the signal $\hat{a}$ to the discontinuity of size $a$ is considered as a random value and associated with a probability density $g_{a}(\hat{a})$. The relation between $a$ and $\hat{a}$ can be expressed as follows:

$$
\hat{a}=\mu(a)+\delta
$$

Here $\mu(a)$ equals the mean value of $g_{a}(\hat{a})$ and $\delta$ is the random error whose distribution determines the probability density $g_{a}(\hat{a})$.

In practice, it is often assumed that $\delta$ is distributed normally with zero mean and constant (independent of $a$ ) variance. $g_{a}(\hat{a})$ is then the normal density function with mean $\mu(a)$ and variance equal to that of $\delta$.

The probability of detection (POD) as function of the size of the discontinuity is:

$$
\operatorname{POD}(a)=P\left\{\hat{a}(a)>\hat{a}_{\mathrm{dec}}\right\}=\int_{\hat{a}_{\mathrm{dec}}}^{+\infty} g_{a}(\hat{a}) d \hat{a}
$$

Fig. 9 illustrates this formula. The probability of detection is represented as hatched part of the area under the bell curve. 
Proc. Vth International Workshop, Advances in Signal Processing for Non Destructive Evaluation of Materials Québec City (Canada),2-4 Aug. 2005. @ X. Maldague ed., É. du CAO (2006), ISBN 2-9809199-0-X

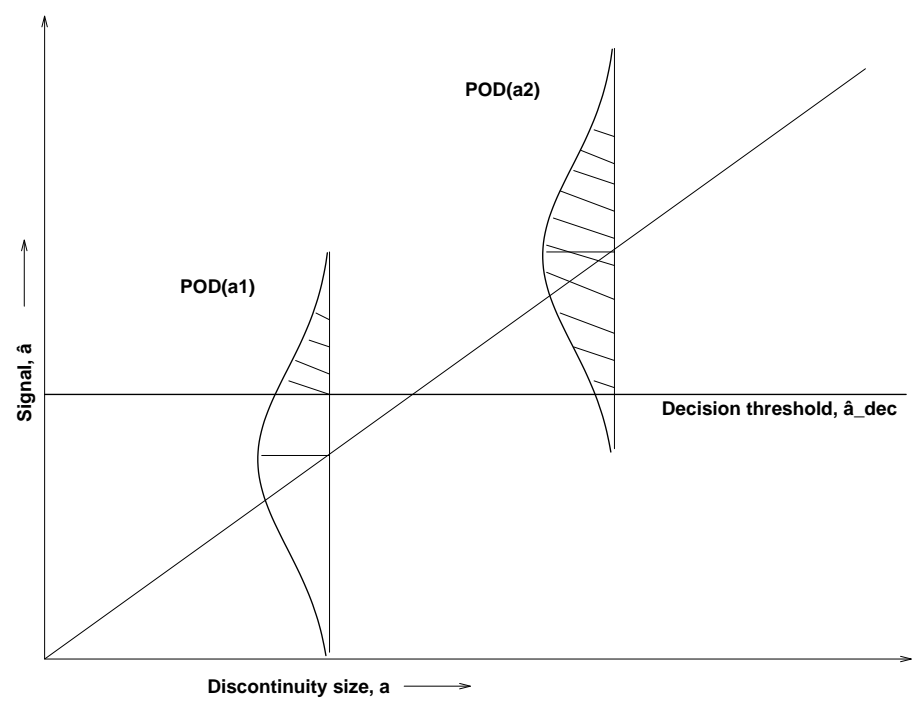

Fig. 9: Probability of detection

\section{Calculation of the POD}

Source data are $a$ and $\hat{a}$ - arrays of length $n$ that contain sizes of the defects and response magnitudes, respectively, and the decision threshold $\hat{a}_{\mathrm{dec}}$. Note that the theory for dealing with censored data has been developed (see [4]) but is not used here, because the data sets available to us do no contain censored data. The censored data are the signals that cannot be registered by the system because they are either under the recording threshold or above the saturation threshold.

\section{Calculation of the POD function parameters}

The following formula is commonly used to model the relation between $a$ and $\hat{a}$ :

$$
\ln \hat{a}=\beta_{0}+\beta_{1} \ln a+\delta
$$

Here $\delta$ is normally distributed with zero mean and constant variance $\sigma_{\delta}^{2}$.

Under the assumptions of the model, the POD function has the following form:

$$
\operatorname{POD}(a)=P\left\{\hat{a}>\hat{a}_{\mathrm{dec}}\right\}=P\left\{\ln (\hat{a})>\ln \left(\hat{a}_{\mathrm{dec}}\right)\right\}=\Phi\left(\frac{\ln a-\mu}{\sigma}\right),
$$

where $\Phi$ is the standard normal distribution function, and

$$
\begin{aligned}
\mu & =\frac{\ln \hat{a}_{\mathrm{dec}}-\beta_{0}}{\beta_{1}} \\
\sigma & =\frac{\sigma_{\delta}}{\beta_{1}}
\end{aligned}
$$


The parameters $\beta_{0}, \beta_{1}$ and $\sigma_{\delta}$ describe the linear dependency of $\hat{a}$ on $a$ and have the following meaning:
$\beta_{0}$
$\beta_{1}$
Intercept
$\sigma_{\delta}$
Slope
Standard deviation of the residuals

Their values are estimated from the arrays $a$ and $\hat{a}$ using the method of maximum likelihood.

\section{The 95\% lower confidence POD}

The $95 \%$ lower confidence bound is given by the following formula:

$$
\operatorname{POD}_{95}(a)=\Phi(\hat{z}-h)
$$

where the variable $h$ reflects the sample size and the scatter of the source data. The calculation of $h$ is thoroughly described in [4].

This general formalism has now to be applied to the defect detection problem within the scope of welding optimization and risk assessment.

\subsection{POD - the original task (full program)}

The original task (together with the welding optimization) is to make sure that only one of 1000 canisters might contain a critical defect situation where in total more or equal $35 \mathrm{~mm}$ of the $\mathrm{Cu}$-wall is missing.

From the naturally real existing POD as a function of all possible influencing parameters we have to extract the POD as function of the flaw radial dimension by a dedicated „Plan of Experiments" and reasonable mean value operations (see Formula 1). The full program is only feasible with a number of additional experiments until the end of 2005. But in order to learn where we are with our current NDT technique and where to optimize, we need a POD assessment of the state of the art using a "Preliminary POD Assessment".

$$
\mathrm{POD}=f\left(a_{1}, \ldots, a_{n}\right) \rightarrow \mathrm{POD}=g\left(a_{\text {radial }}\right)
$$

Formula 1: Extraction of the POD as a function of the radial size.

\section{3 „Preliminary POD assessment"}

Volumetric flaws and area-like (non-volumetric) flaws will be treated separately - for EB as well as for FSW

- Volumetric flaws RT

- area like flaws UT 
Proc. Vth International Workshop, Advances in Signal Processing for Non Destructive Evaluation of Materials Québec City (Canada),2-4 Aug. 2005. ( X. Maldague ed., É. du CAO (2006), ISBN 2-9809199-0-X

The physically reasonable „, $\hat{a}$ versus $a^{\prime \prime}$ POD including physically reasonable 2D extensions will be applied to the parameter configurations shown in the Table 1.

\begin{tabular}{|l|l|l|}
\hline Method & Parameter & Physical meaning \\
\hline \multirow{2}{*}{ RT } & $a$ & penetrated length of a discontinuity \\
\cline { 2 - 3 } & $\hat{a}$ & maximum contrast \\
\hline \multirow{2}{*}{ UT } & $a$ & area of a discontinuity perpendicular to the sound beam \\
\cline { 2 - 3 } & $\hat{a}$ & maximum echo height \\
\hline
\end{tabular}

Table 1. Parameter configurations for the POD calculation.

We determine the $a_{90 / 95}$ magnitudes i.e. the size $a$ of the flaw for which the lower $95 \%$ confidence bound crosses the $90 \%$ POD level i.e. it is guaranteed that flaws with a size of $a_{90 / 95}$ will be detected with $90 \%$ probability where only $5 \%$ might fall outside this confidence limit in case the experiment is repeated. We work with the assumption - given by the manufacturing experts -- that only one of 100 canisters might have a critical flaw. Then the above argumentation yields: Only one of 1000 canisters might have a leakage.

\subsection{Integrity requirement}

The flaw radial size has to be limited so that a remaining wall thickness of $15 \mathrm{~mm}$ is guaranteed against ground water corrosion. The maximum allowed flaw size in the radial direction is $35 \mathrm{~mm}$. Together with the above POD an additional flaw geometry statistic has to provide for the four groups

- FSW volumetric area-like

- EB volumetric area-like

... separately, no flaws of $a_{\mathrm{r}} \geq 35 \mathrm{~mm}$ will be present. No flaw of radial size $a_{\mathrm{r}} \geq 35 \mathrm{~mm}$ can be among the flaw assembly below $a_{90 / 95}$, that means no $a \leq a_{90 / 95}$ should have at the same time an $a_{\mathrm{r}} \geq 35 \mathrm{~mm}$. This would yield a reasonable justification of the system to meet the above integrity requirement, as long as the existing flaw configurations are representative for the welds. Look for the "correlation" in scatter diagrams:

- $\quad$ penetrated flaw length by X-ray $\leftarrow \rightarrow$ radial dimension

- $\quad$ area perpendicular to UT beam $\leftarrow \rightarrow$ radial dimension 
Proc. Vth International Workshop, Advances in Signal Processing for Non Destructive Evaluation of Materials Québec City (Canada),2-4 Aug. 2005. @ X. Maldague ed., É. du CAO (2006), ISBN 2-9809199-0-X

\subsection{Results}

\section{Volumetric flaws}

In the following diagrams we illustrate the so called preliminary results for the examples of volumetric flaws for EBW and FSW detected by X-rays.

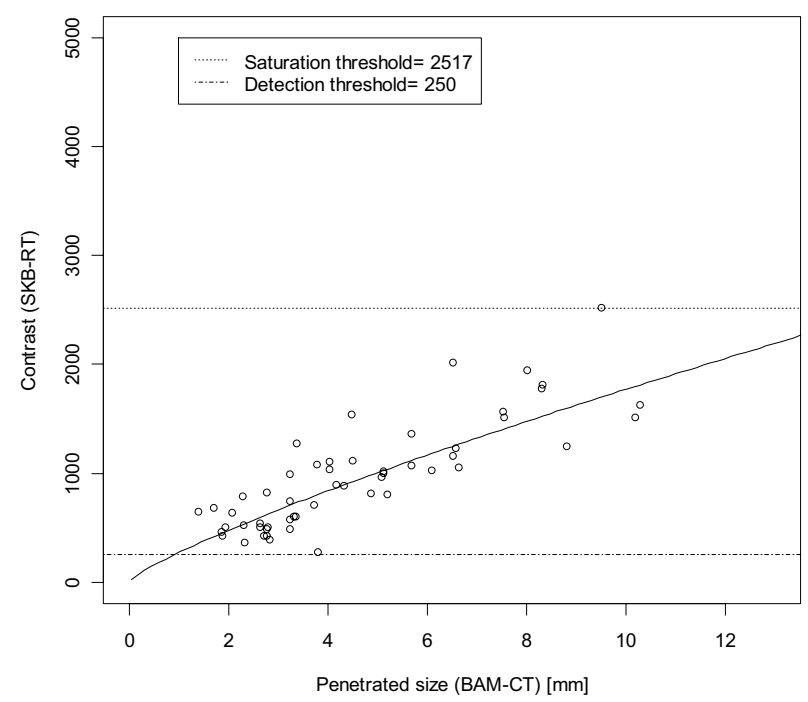

Fig. 10: " $\hat{a}$ vs. $a$ " diagram of the contrast vs. penetrated size (electron beam welding, radiographic inspection, tomographic reference inspection)

Fig. 10 shows the basic " $\hat{a}$ versus $a$ " diagram for the radiographic maximum contrast as a function the defect size penetrated by the X-rays. The dependence is quite linear but shows a considerable scatter.

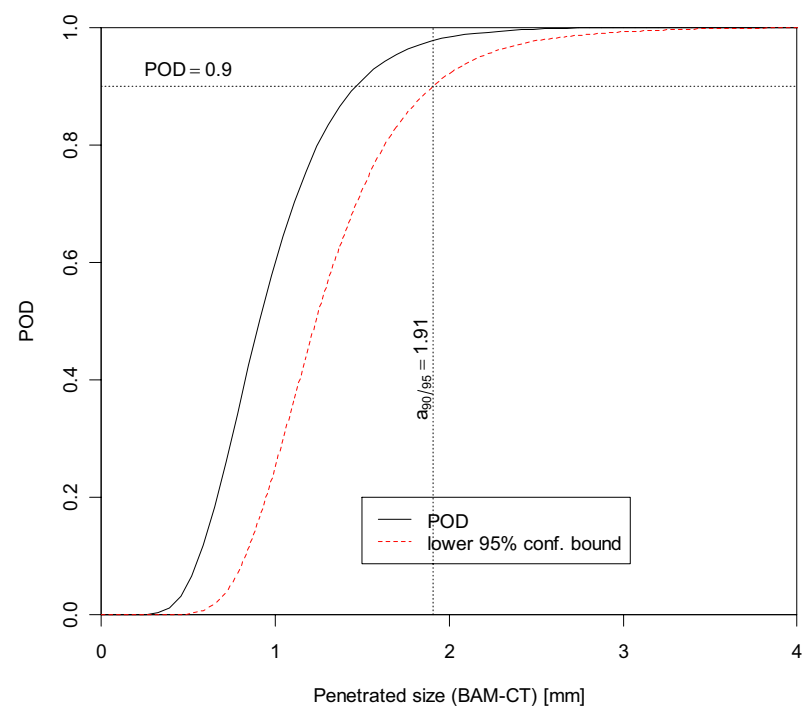

Fig. 11: "Probability of detection" curve (electron beam welding, radiographic inspection, tomographic reference inspection) 
The corresponding probability of detection and its lower confidence bound (Fig. 11) yield a $a_{90 / 95}$ value of about $2 \mathrm{~mm}$ for the penetrated size. Now we have to make sure the corresponding radial dimension will not exceed the critical size for all penetrated sizes below $a_{90 / 95}$.

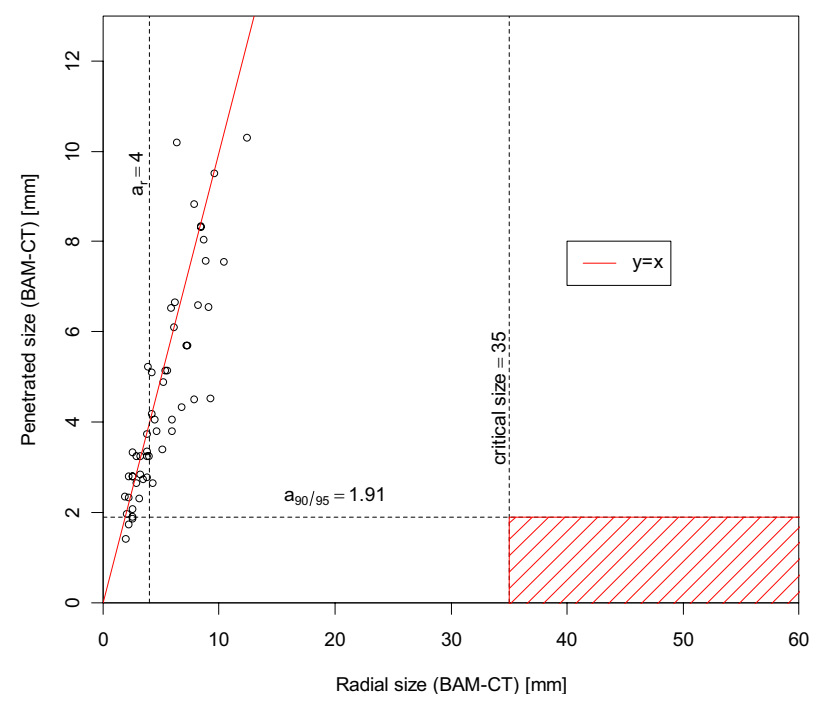

Fig. 12: Scatter diagram of the penetrated size vs. radial size with the critical region (electron beam welding, radiographic inspection, tomographic reference inspection)

Fig. 12 shows the defect size statistics where the penetrated sizes are plotted against the radial dimensions for all observed volumetric flaws for the EBW. The critical region is the red hatched area, where defects with critical radial dimensions above the critical size would occur belonging to sizes $a$ below $a_{90 / 95}$. As seen in the Fig. 12, the real defect configurations are far away from this.

The Fig. 13 to Fig. 15 show the same types of diagrams for the X-ray investigation of volumetric flaws for FSW. The scatter of signals is wider and the $a_{90 / 95}$ is a bit bigger. But again all observed defects are far away from the critical region. 
Proc. Vth International Workshop, Advances in Signal Processing for Non Destructive Evaluation of Materials Québec City (Canada),2-4 Aug. 2005. @ X. Maldague ed., É. du CAO (2006), ISBN 2-9809199-0-X

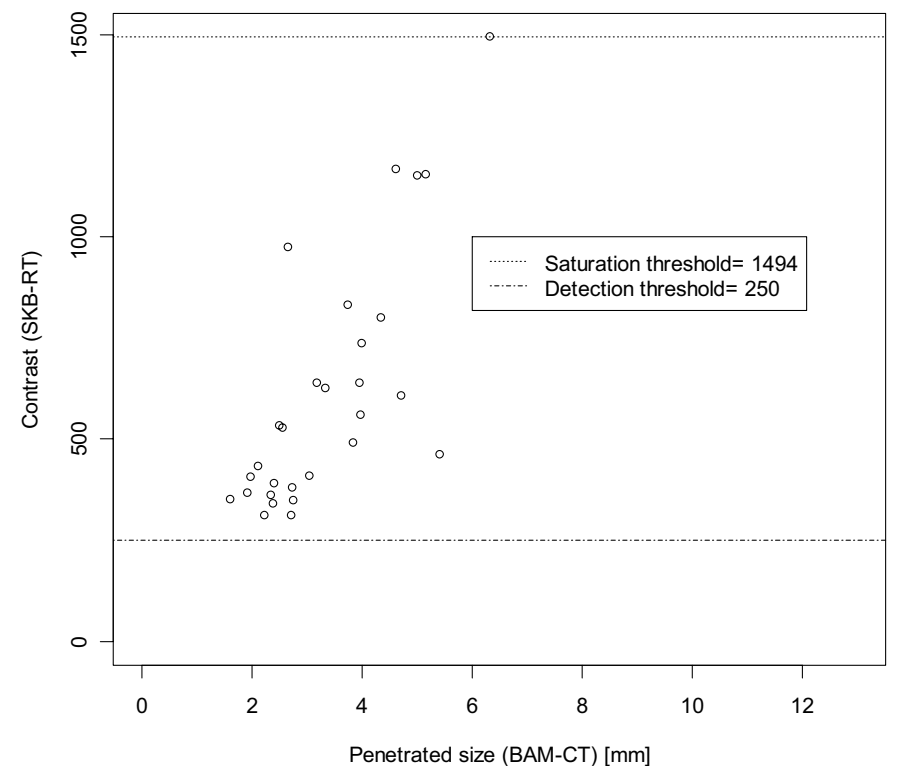

Fig. 13: " $\hat{a}$ vs. $a$ " diagram of the log. contrast vs. penetrated size (friction stir welding, radiographic inspection, tomographic reference inspection)

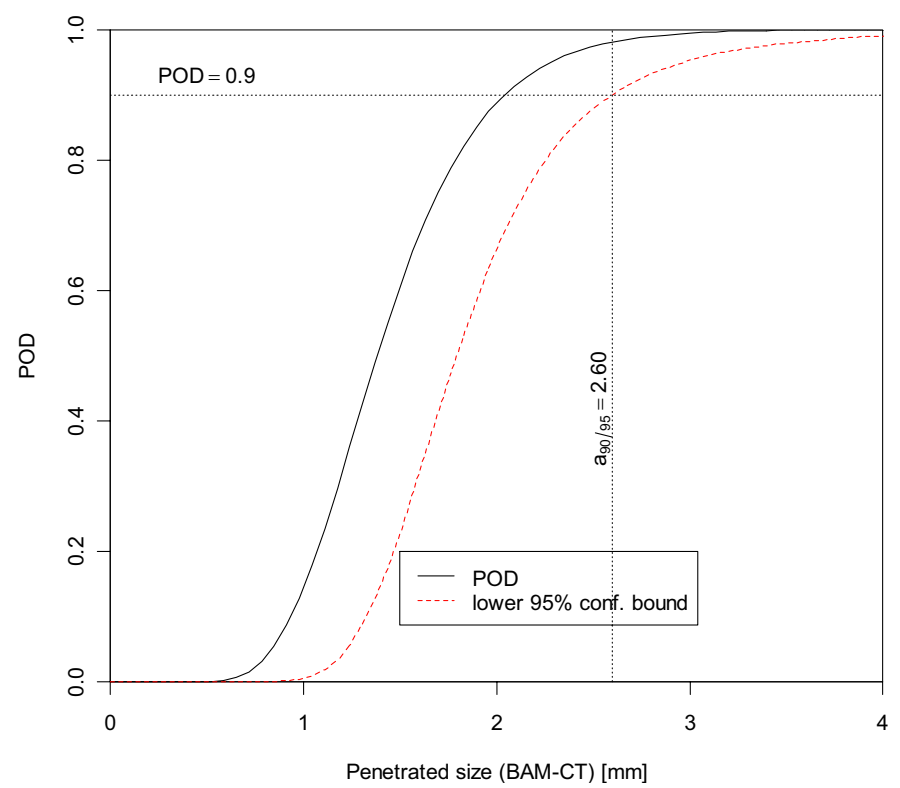

Fig. 14: "Probability of detection" curve (friction stir welding, radiographic inspection, tomographic reference inspection 
Proc. Vth International Workshop, Advances in Signal Processing for Non Destructive Evaluation of Materials Québec City (Canada),2-4 Aug. 2005. @ X. Maldague ed., É. du CAO (2006), ISBN 2-9809199-0-X

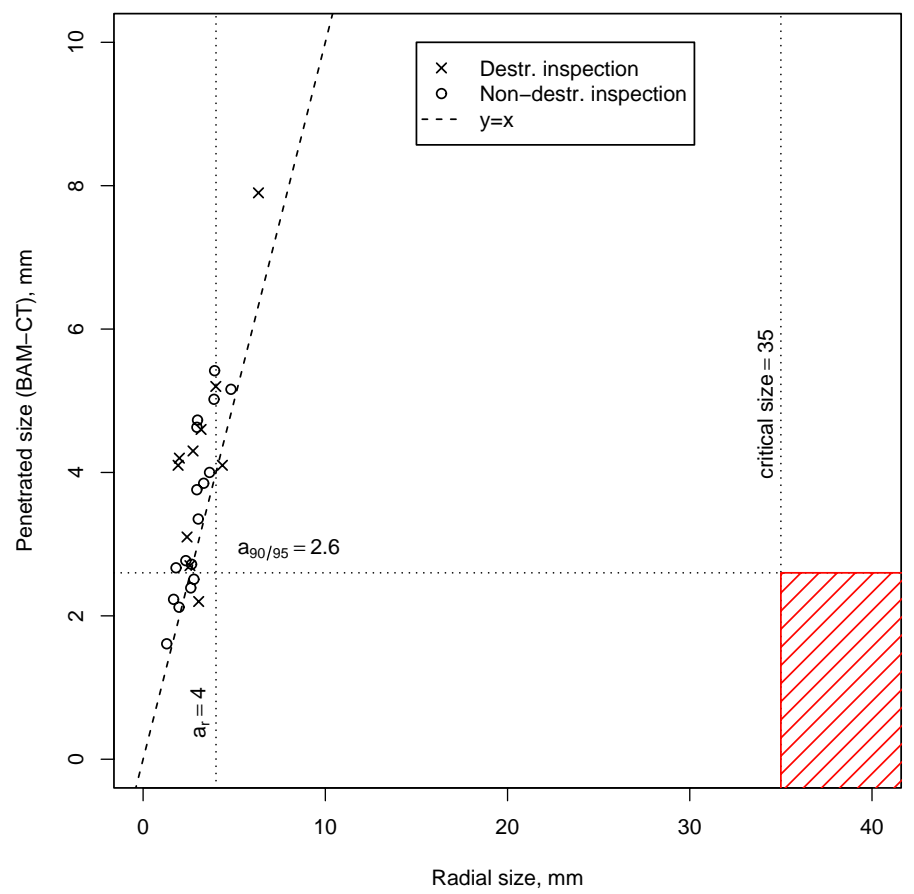

Fig. 15: Scatter diagram of the penetrated size vs. radial size with the critical region (friction stir welding, radiographic inspection, tomographic reference inspection)

\section{Joint line hooking}

Another relevant type of flaws occurring in the friction stir welds are the so called joint line hooking (JLH) flaws. An example photo showing the cross section of such a flaw is presented on Fig. 16.

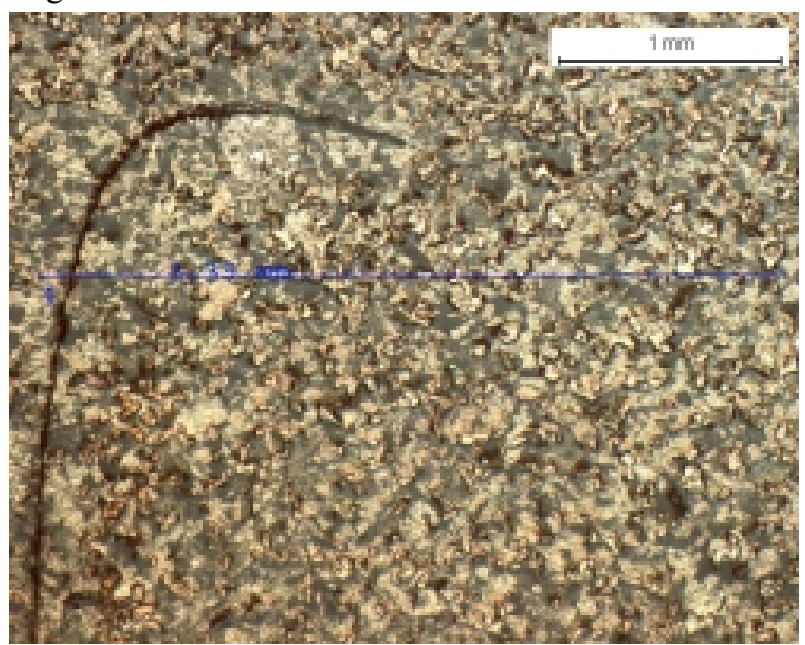

Fig. 16: Cross section of a JLH (Joint Line Hooking)

The first approach in investigating the JLH flaws was to apply the POD method in an empirical way. For $\hat{a}$ we applied the maximum echo amplitude as before. First we created a 
Proc. Vth International Workshop, Advances in Signal Processing for Non Destructive Evaluation of Materials Québec City (Canada),2-4 Aug. 2005. @ X. Maldague ed., É. du CAO (2006), ISBN 2-9809199-0-X

totally empirical " $\hat{a}$ versus $a$ " scatter diagram (Fig. 17) and POD (Fig. 18) with all the values of the SKB experiments included which yields the $a_{90 / 95}$ equal to the detectable radial dimension of $4.0 \mathrm{~mm}$ based on a threshold of $6 \%$ screen height. When we exclude outliers (too small and too big values compared to a "normal" amplitude versus size behavior the detectable radial dimension is $3.2 \mathrm{~mm}$ because the scatter is diminished.

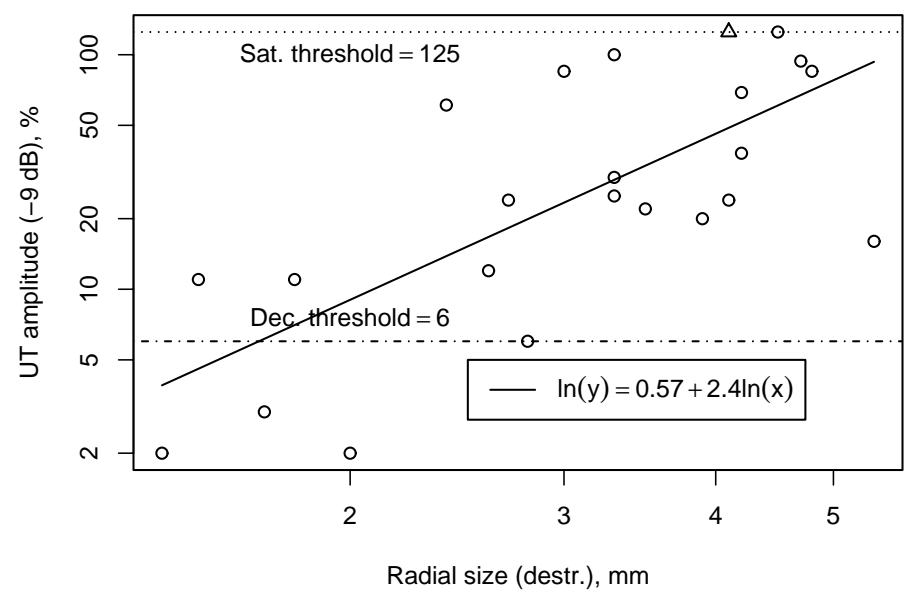

Fig. 17: Scatter diagram of the amplitude vs. radial size for the JLH-type flaws (log. axes).

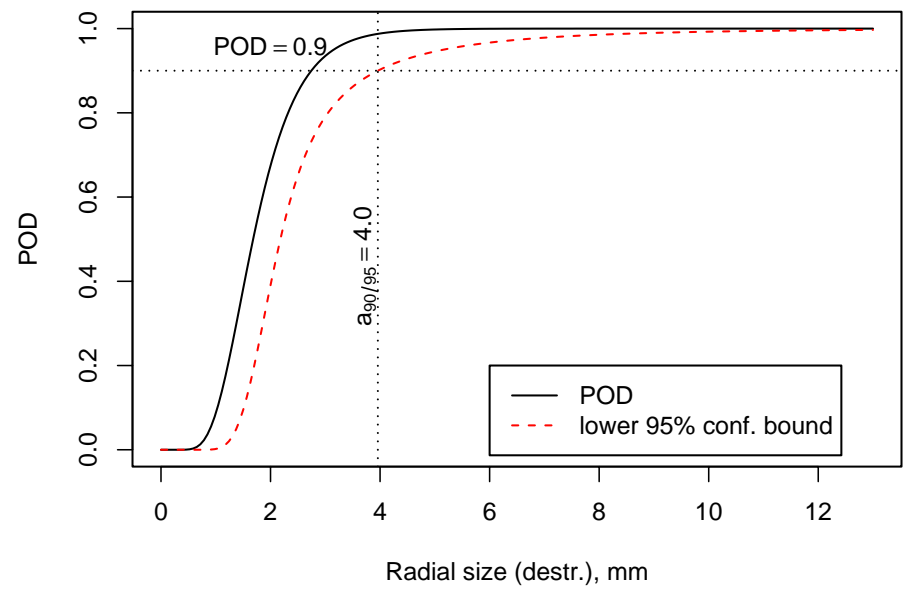

Fig. 18: POD curve for the JLH-type flaws (with outliers). 


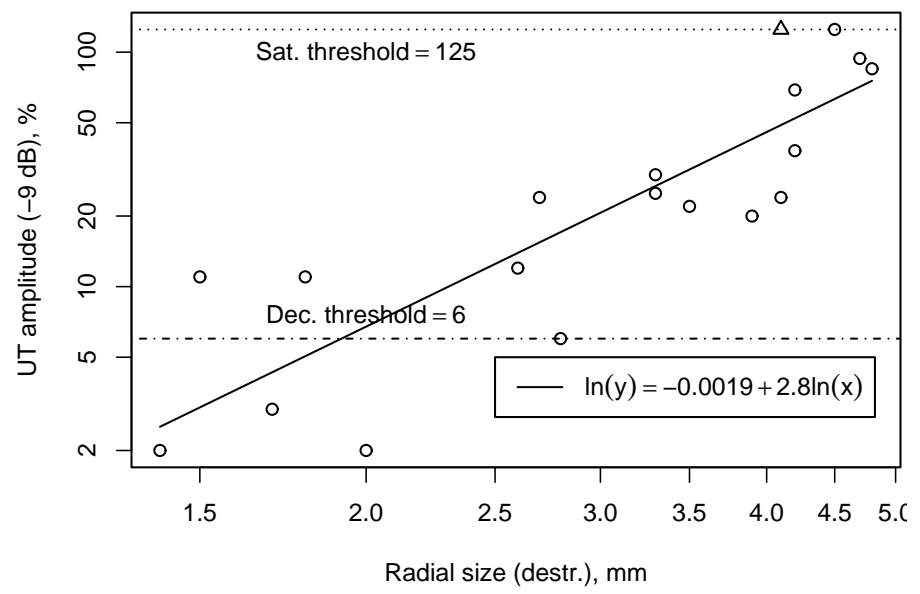

Fig. 19: Scatter diagram of the amplitude vs. radial size for the JLH-type flaws (log. axes, without outliers).

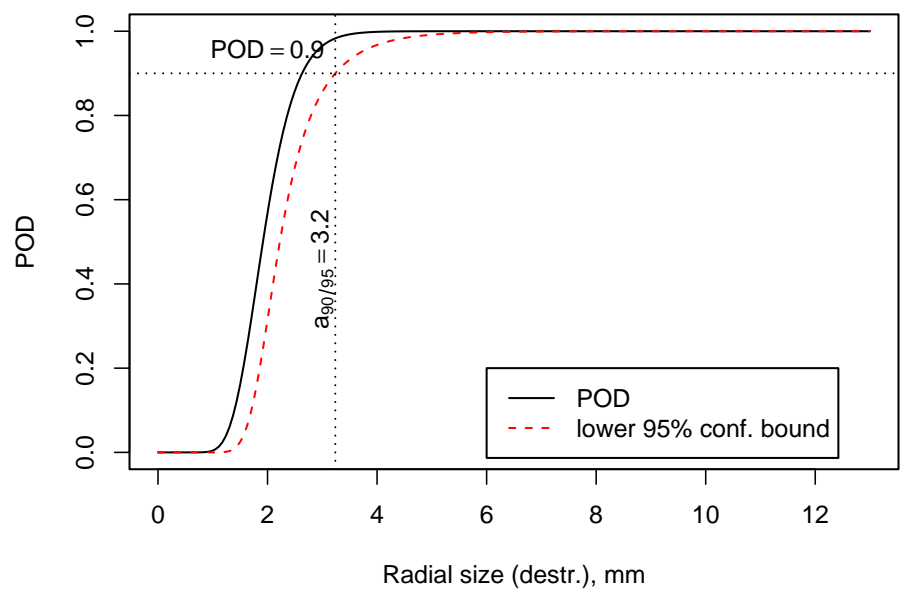

Fig. 20: POD curve for the JLH-type flaws (outliers excluded).

The evaluation of the amplitude data concerning the JLH discontinuities together with the macrographs gave some new information. The reflectivity of the joint line hooking discontinuities (JLH) is not independent from the angle of incidence, as if a side drilled hole were under investigation. This is caused by the geometry of the discontinuity (e.g. radius of curved part, inclination of planar part) and the reflectivity of the discontinuity's surface (e.g. caused by the kissing bond effect). This provides the motivation for separating the whole flaw into regions having different geometrical and reflective properties and being inspected under varying incidence angles. The separation is shown on

Fig. 21.

As the number of data points becomes too small after separation, an UT simulation tool has been used to approximate both experimental situations.

The hook and rectangle parts have been modelled by curved rectangles (sections of cylindrical surface) and flat rectangles, accordingly. Varying curvature radii, radial widths, inclination angles and incidence angles have been considered. 
Proc. Vth International Workshop, Advances in Signal Processing for Non Destructive Evaluation of Materials Québec City (Canada),2-4 Aug. 2005. @ X. Maldague ed., É. du CAO (2006), ISBN 2-9809199-0-X

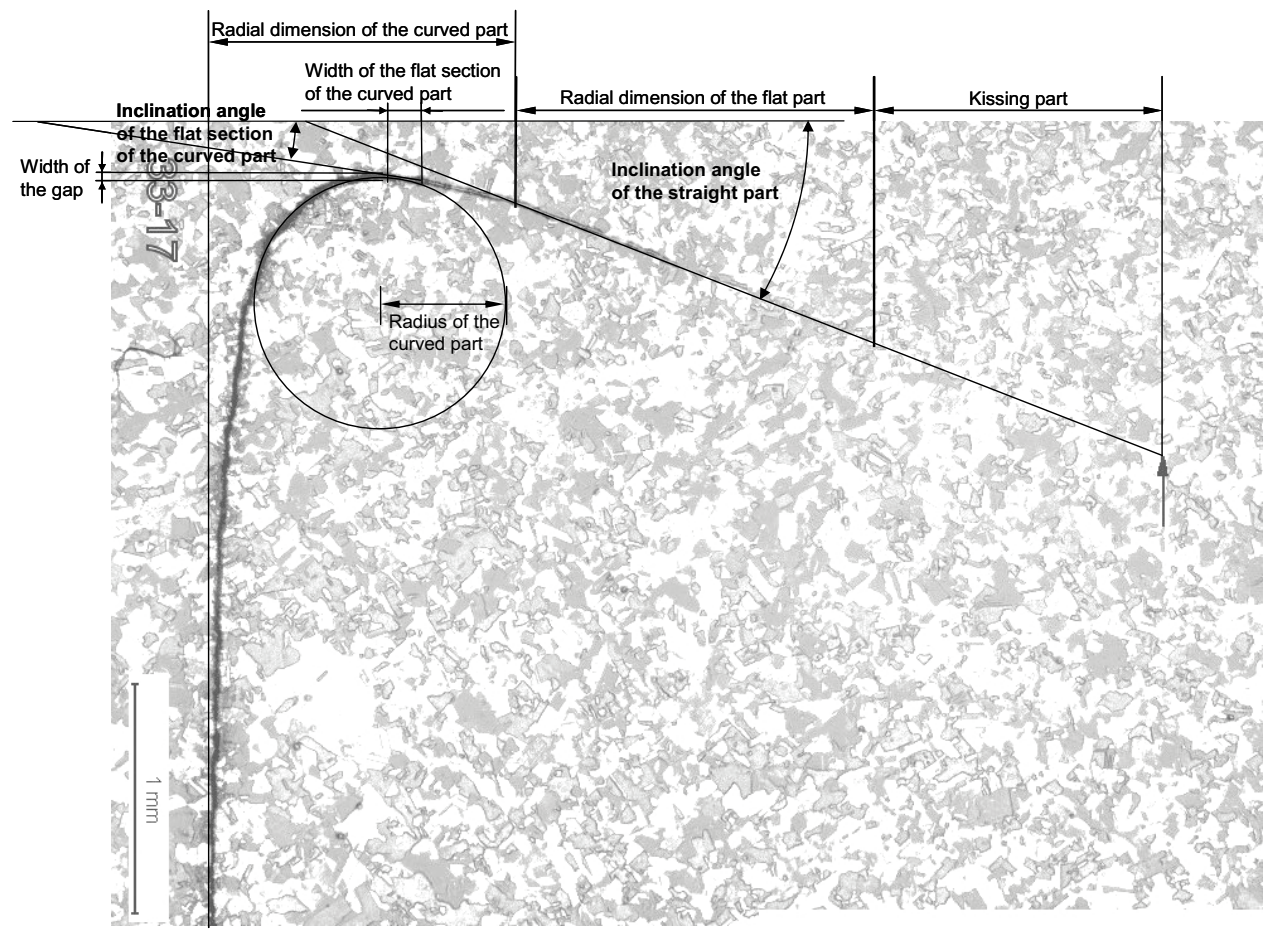

Fig. 21: Regions of the JLH flaws.

An example scatter diagram for a simulated rectangle having the inclination angle of 70 degrees and being inspected under the incidence angle of 20 degrees is shown on Fig. 22, and the corresponding POD curve on Fig. 23.

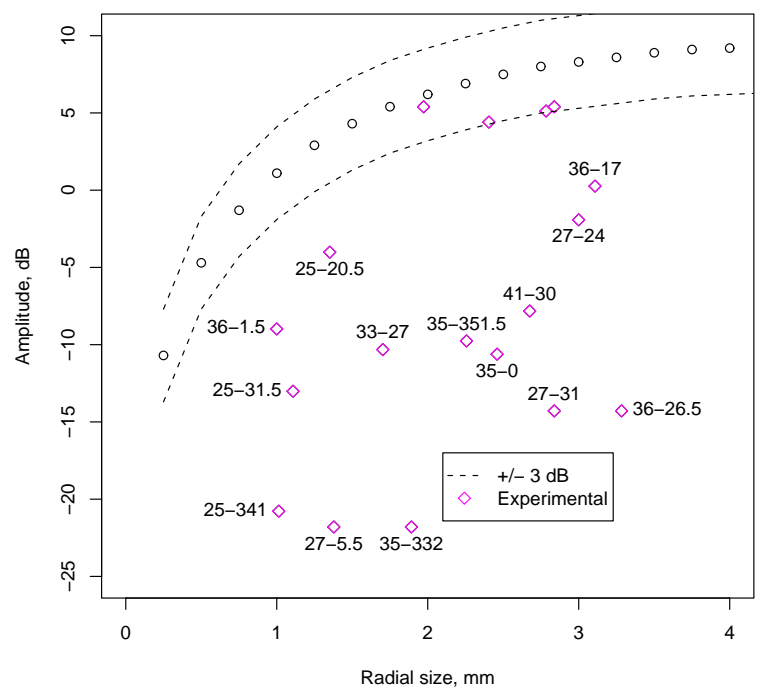

Fig. 22. Simulated scatter diagram (with experimental points representing corresponding parts of real discontinuities) of the amplitude vs. radial size for a simulated rectangle (inclination angle $70 \mathrm{deg}$. incidence angle $20 \mathrm{deg}$.). 
Proc. Vth International Workshop, Advances in Signal Processing for Non Destructive Evaluation of Materials Québec City (Canada),2-4 Aug. 2005. @ X. Maldague ed., É. du CAO (2006), ISBN 2-9809199-0-X

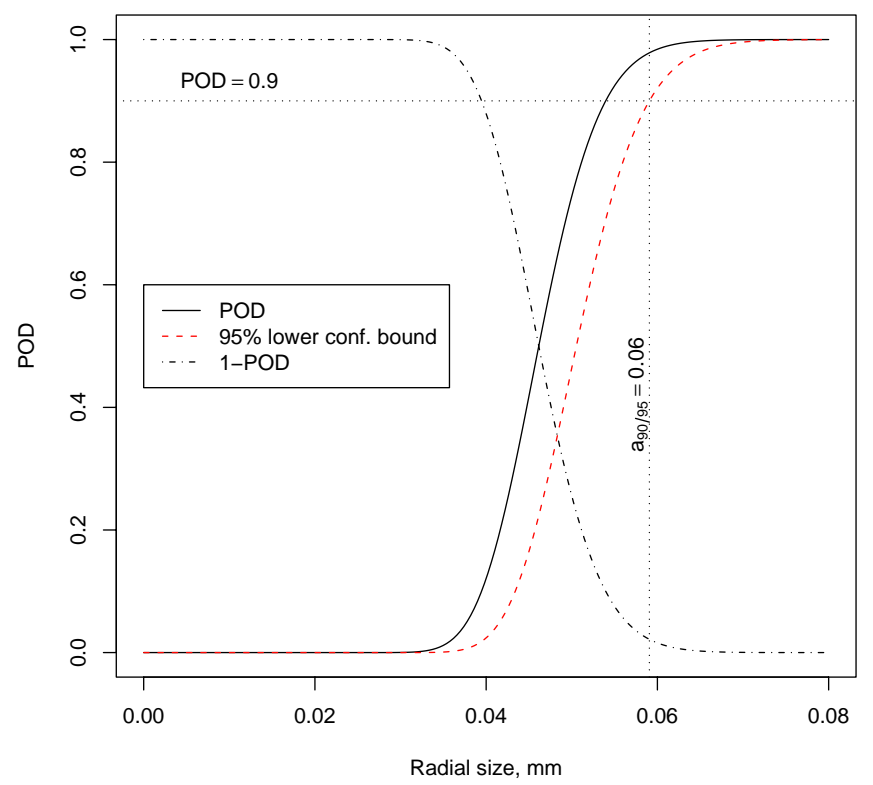

Fig. 23. POD curve based on simulated data for a rectangle (inclination angle $70 \mathrm{deg}$, incidence angle 20 deg.).

We would like to emphasize here that the modelling background helps to verify that both parts of the JLH are good ultrasonic reflectors, which is confirmed by the model. A greater coincidence between model and experiment can be reached from both sides:

i) to create a more realistic shaped model in the computer for calculation and

ii) to create a physical model with the shape closer to the ideal one for template experiments.

Important for the safety consideration is the straight part of the JLH which might grow towards the welding surface. The modelled POD results yield a $a_{90 / 95}$ below $1 \mathrm{~mm}$ which seems to be too optimistic since the modelled echo curve covers the experimental values from the higher side. For further studies it should be analysed systematically what causes the differences in the echo heights. 


\subsection{Consideration of the total reliability}

The PODs considered so far reveal the basic, or intrinsic capability of the NDT methods and do not yet take into account the final industrial application factors and the human factor. The situation for general NDT systems - as outlined in the formula in Formula 2- is described in detail in [5] and [6] and will be applied to the canister welds on a later stage of the project.

$$
R \equiv f(\mathrm{IC})-g(\mathrm{AP})-h(\mathrm{HF})
$$

\section{Formula 2: Modular approach: the reliability formula}

The following table explaing the meaning of the symbols in Formula 2.

$\mathrm{R} \quad$ Total reliability of an NDE system or mine searching system

IC Intrinsic capability of the system driven by physical laws and technical potential generally considered as an ideal upper bound

AP The effect of industrial application parameters, such as access restrictions, surface state, generally reducing the capability of the ideal NDE system

HF The effect of human factors, often further reducing the capability of the NDE system

\section{Result}

As result of this SKB-BAM project optimized testing techniques will be provided which are validated for the production process and which will guarantee the required rest wall thickness with sufficient reliability. The joint SKB-BAM project is a contribution for the long term European public safety.

\section{Acknowledgment}

The authors like to thank Dr. Gerd_Rüdiger Jaenisch for fruitful scientific discussions and Dr. Jürgen Goebbels for providing the reference - CT-measurements on "High energy - CT". We are also indebted to the colleagues from the ultrasonic group at BAM (Dr. Gerhard Brekow, Dr. Dirk Tscharntke) for ultrasonic reference measurements and Mrs. Sylke Bär for helpful assistance in flaw extraction from data sets. 


\section{References}

[1] X. X. RD \& D-programme 2001. Technical Report TR-01-30, Svensk Kärnbränslehantering AB, September 2001.

[2] Gerd-Rüdiger Tillack, Christina Nockemann, and Carsten Bellon. X-ray modelling for industrial applications. NDT \& E International, 33:481-488, 2000.

[3] US Department of defense. Nondestructive evaluation system. Reliability assessment, 1999. Handbook.

[4] Alan P. Berens. NDE reliability data analysis. In Metals Handbook, volume 17. ASM International, 9 edition, 1989.

[5] Christina Müller, Thomas Fritz, Gerd-Rüdiger Tillack, Carsten Bellon, and Martina Scharmach. Theory and applications of the modular approach to NDT reliability. Materials Evaluation, 59(7):871-874, 2001.

[6] Christina Müller, Mstislav Elaguine, Martina Scharmach, Bernhard Redmer, Uwe Ewert, Lloyd Schaefer, and Peter-Theodor Wilrich. Reliability investigation of NDE systems by modular analysis of recorded data. In Proceedings of the 8th European Conference on Non-Destructive Testing, 2002. 
Proc. Vth International Workshop, Advances in Signal Processing for Non Destructive Evaluation of Materials Québec City (Canada),2-4 Aug. 2005. @ X. Maldague ed., É. du CAO (2006), ISBN 2-9809199-0-X

\section{Correspondence Address}

Dr. rer. nat., ORRin Christina Müller

postal adress:

Bundesanstalt für Materialforschung und - prüfung,

Unter den Eichen 87, 12205 Berlin, Germany

telefone: $\quad+49-3081041833$

fax number: $\quad$ +49-30 81041836

e-mail adress: Christina.Mueller@bam.de 